\title{
The association of personal and neighborhood socioeconomic indicators with subclinical cardiovascular disease in an elderly cohort. The cardiovascular health study is
}

\author{
Cheryl K. Nordstrom ${ }^{\mathrm{a}, *}$, Ana V. Diez Roux ${ }^{\mathrm{a}}$, Sharon A. Jackson ${ }^{\mathrm{b}}$, \\ Julius M. Gardin ${ }^{c, d}$ \\ a Department of Epidemiology, School of Public Health, University of Michigan, 1214 S. 2nd Floor, Ann Arbor, MI 48104-2548, USA \\ ${ }^{\mathrm{b}}$ Department of Public Health Sciences, Wake Forest University School of Medicine, Medical Center Boulevard, Winston-Salem, \\ NC 27107, USA

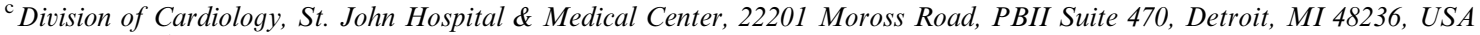 \\ ${ }^{\mathrm{d}}$ Department of Internal Medicine, Wayne State University School of Medicine, Detroit, MI, USA
}

\begin{abstract}
There has been recent interest in determining whether neighborhood characteristics are related to the cardiovascular health of residents. However, there are no data regarding the relationship between neighborhood socioeconomic status (SES) and prevalence of subclinical cardiovascular disease (CVD) in the elderly. We related personal SES (education, income, and occupation type) and neighborhood socioeconomic characteristics (a block-group score summing six variables reflecting neighborhood income and wealth, education, and occupation) to the prevalence of subclinical CVD (asymptomatic peripheral vascular disease or carotid atherosclerosis, electrocardiogram or echocardiogram abnormalities, and/or positive responses to Rose Questionnaire claudication or angina pectoris) among 3545 persons aged 65 and over, without prevalent CVD, in the Cardiovascular Health Study. Sixty percent of participants had at least one indicator of subclinical disease. Compared to those without, those with subclinical disease had significantly lower education, income, and neighborhood scores and were more likely to have blue-collar jobs. After adjustment for age, gender, and race, those in the lowest SES groups had increased prevalence of subclinical disease compared with those in the highest SES groups ( $\mathrm{OR}=1.50 ; 95 \% \mathrm{CI} 1.21,1.86$ for income; $\mathrm{OR}=1.41 ; 95 \% \mathrm{CI} 1.18,1.69$ for education; $\mathrm{OR}=1.39 ; 95 \%$ CI 1.16, 1.67 for block-group score). Those reporting a blue-collar lifetime occupation had greater prevalence of subclinical disease relative to those reporting a white-collar occupation ( $\mathrm{OR}=1.29 ; 95 \% \mathrm{CI} 1.02-1.59)$. After adjustment for behavioral and biomedical risk factors, all of these associations were reduced. Neighborhood score tended to remain inversely associated with subclinical disease after adjustment for personal socioeconomic indicators but associations were not statistically significant. Personal income and blue-collar occupation remained significantly associated with subclinical disease after simultaneous adjustment for neighborhood score and education. Personal and neighborhood socioeconomic indicators were associated with subclinical disease prevalence in this elderly cohort. These relationships were reduced after controlling for traditional CVD risk factors.
\end{abstract}

(C) 2004 Elsevier Ltd. All rights reserved.

Keywords: Socioeconomic status; Income; Education; Occupation; Subclinical disease

\footnotetext{
The research reported in this article was supported by contracts N01-HC-85079-N01-HC-85086 from the National Heart, Lung, and Blood Institute, and Georgetown Echo RC-HL 35129 JHU MRI RC-HL 15103, and by R29HL59386.

*Corresponding author. Tel.: + 734-763-2303; fax: + 734-998-0006.

E-mail address: nrdstrom@umich.edu (C.K. Nordstrom).
} 


\section{Introduction}

Socioeconomic status (SES) has been extensively reviewed in relation to the risk of cardiovascular disease (CVD) events (Kaplan \& Keil, 1993). Multiple studies have shown inverse associations between socioeconomic position and prevalence and incidence of CVD (Rose \& Marmot, 1981; Feldman et al., 1989; Marmot et al., 1991; Diez-Roux, Nieto, Tyroler, Crum, \& Szklo, 1995). Recent work has also shown that living in disadvantaged neighborhoods is associated with increased coronary heart disease even after accounting for personal socioeconomic indicators (Diez-Roux et al., 2001b).

There are multiple processes through which personal and neighborhood socioeconomic characteristics could affect CVD risk. For example, those with lower education levels may not be fully aware of the consequences of eating a diet high in saturated fat or trans-fatty acids or may not be able to afford a healthy diet. Those in disadvantaged neighborhoods may not have access to fresh fruits and vegetables, or they may not have access to safe places to walk (physical activity) or otherwise exercise. Those with blue-collar jobs may face social norms at the workplace that support smoking, a CVD risk factor more common in bluecollar job sites. Persons in poverty or facing economic adversity may experience greater stresses associated with not being able to pay bills, not feeling safe in their homes, etc., which may increase catecholamine responses or other hormones that adversely affect CVD profiles.

Personal SES has also been related to carotid intimamedia thickness (IMT), an indicator of early, subclinical atherosclerotic disease (Diez-Roux et al., 1995; Lynch, Kaplan, Salonen, Cohen, \& Salonen, 1995; Lynch, Kaplan, Salonen, \& Salonen, 1997; Lamont et al., 2000; Rosvall et al., 2000). All of these studies found protective effects of high SES on either IMT or its progression. However, findings differed across SES indicators at the individual level (education, income, occupation), or across measures of IMT (mean, maximum, plaque height, carotid stenosis) (Diez-Roux et al., 1995; Lynch et al., 1995, 1997; Lamont et al., 2000; Rosvall et al., 2000). Data relating neighborhood SES indicators and carotid IMT have not been published.

The purpose of this study was to investigate the crosssectional relationships between both personal SES (education, income, job classification) and neighborhood SES (a score for participant's geographic blockgroup based on area wealth and income) and prevalent subclinical CVD in a cohort of elderly men and women in the Cardiovascular Health Study (CHS). Subclinical disease measures have been shown to be predictive of future clinical events (Salonen \& Salonen, 1993; Bots et al., 1997; Chambless et al., 1997; Hodis et al., 1998; O'Leary et al., 1999). Thus, the investigation of factors associated with early disease is of interest from the point of view of disease prevention. The examination of associations between neighborhood characteristics and measures of subclinical disease also avoids biases associated with the migration of clinically ill persons into poorer neighborhoods. In addition, to the extent that neighborhood characteristics are correlated over a person's lifetime, the investigation of associations in an elderly cohort allows quantification of the relationship between neighborhood characteristics and a summary measure of the extent of atherosclerosis developed over the lifecourse. Potential mediators and confounders of these relationships were also examined.

\section{Methods}

\section{Study population and study variables}

The CHS is a population-based longitudinal study of coronary heart disease and stroke in adults aged 65 years and older (Fried et al., 1991). In 1989-1990, 5201 men and women were recruited from Medicare eligibility lists in four communities: Forsyth County, North Carolina; Sacramento County, California; Washington County, Maryland; and Pittsburgh, Pennsylvania. In 1992-1993, an additional 687 black participants from three of these four geographic locations (excluding Washington County, MD) were recruited into the study.

The outcome for this study, collected at the baseline examination, was the composite index of subclinical atherosclerosis and CVD developed in the CHS (Kuller et al., 1994). Briefly, any one or more of the following criteria was classified as prevalent subclinical disease: ankle-arm index $\leqslant 0.90$, internal carotid wall thickness $>80$ th percentile, common carotid wall thickness $>80$ th percentile, carotid stenosis $>25 \%$, major electrocardiogram abnormalities, abnormal ejection fraction on echocardiogram, abnormal wall motion on echocardiogram, Rose Questionnaire positive for claudication, or Rose Questionnaire positive for angina pectoris. The cohort recruited in 1992-1993 did not undergo echocardiographic assessment, therefore, classification of subclinical disease for these participants does not include ejection fraction or wall motion.

Personal SES information was obtained from the baseline examination interview. Educational attainment was reported to the interviewer and was scored from 0 to 21 based on the following categories: no schooling $($ score $=0)$; grades 1-12 ( tional Development test $($ score $=13) ; 1,2$, or 3 years of vocational training $($ score $=14-16) ; 1,2,3$, or 4 years of college $($ score $=17-20)$; graduate or professional school $($ score $=21)$. For the analyses, education was modeled as an ordinal categorical variable based on three education 
groups (high school or less, some college or vocational training, completed college or graduate school).

Occupation was defined as usual lifetime occupation and was selected from a response card as one of the following: professional/technical/managerial/administrative; sales/clerical service; craftsman/machine operator/laborer; farming/forestry; housewife; other. Responses in the farming/forestry and craftsman/machine operator/laborer category were classified as having a "blue-collar occupation" for purposes of these analyses. Those reporting housewife, other, or refused were not included in the occupational analyses, which were based on a dichotomous variable for blue collar "yes" or "no". Personal income was defined as total family income before taxes from all sources in the past 12 months and was selected from a response card as one of the following: under $\$ 5000$; $\$ 5000-\$ 7999 ; \$ 8000$ $\$ 11,999 ; \quad \$ 12000-\$ 15999 ; \quad \$ 16,000-\$ 24,999 ; \quad \$ 25,000$ $\$ 34,999$; $\$ 35,000-\$ 49,999$; over $\$ 50,000$. For the analyses, income was modeled as an ordinal categorical variable based on three income groups $(<\$ 12,000$, $\$ 12,000-\$ 34,999$, $\geqslant \$ 35,000)$.

Neighborhood socioeconomic characteristics were summarized in a neighborhood score (BGScore). Census-defined block-groups were used as proxies for neighborhoods. The score was constructed by combining six variables derived from 1990 US Census data for block-groups based on the home address the participant reported at the baseline examination (Diez-Roux et al., 2001a,b). Variables included in the summary score represent neighborhood income and wealth $(\log$ of the median household income, log of the median value of housing units, and the percentage of households receiving interest income); neighborhood education (the percentage of adults 25 years of age or older who had completed high school and the percentage of adults 25 years of age or older who had completed college); and neighborhood occupation (the percentage of employed persons 16 years of age or older in executive, managerial, or professional specialty occupations). For each variable, a $z$-score, reflecting the deviation of the value from the mean across all block groups in the sample, was calculated. The sum of the $z$-scores for each of the six variables was deemed the neighborhood summary score. Block-group score in this sample ranged from -10.7 to 16.7 , with a higher score reflecting a higher SES for the neighborhood. For the analyses, neighborhood SES score was modeled as an ordinal categorical variable based on tertiles of this score.

Additional covariates were collected from the baseline examination interview and physical examinations. Selection of covariates for these analyses was guided by analyses of correlates of subclinical CVD in the CHS by Kuller et al. (1994), and includes age, gender, race, use of cigarettes and alcohol, physical activity (total kilocalories of activity performed per week), use of anti- hypertensive medication, systolic blood pressure (SBP), diastolic blood pressure (DBP), body mass index (BMI), total serum cholesterol, high-density lipoprotein cholesterol (HDL), fasting blood glucose, and American Diabetes Association diabetes status.

Of 5888 participants at baseline, 5174 could be matched to block groups with available census data. Of these, 47 were excluded because they matched block groups with less than 100 persons, less than 30 housing units, and/or $33 \%$ or more persons living in group quarters. Because we were interested in examining associations with subclinical disease, an additional 1569 participants with clinical CVD at baseline (atrial fibrillation or pacemaker or history of intermittent claudication, peripheral vascular disease, congestive heart failure, stroke, transient ischemic attack, carotid surgery, coronary artery bypass graft, percutaneous transluminal coronary angioplasty, angina, use of nitroglycerin, or myocardial infarction) were excluded, as were 22 persons who identified themselves as being neither black nor white, leaving 3545 participants available for these analyses.

\section{Statistical analyses}

Means and percentage distributions of SES indicators and covariates for those with and without subclinical disease were calculated. Student's $t$-test and chi-square test were used to assess the significance of the betweengroup differences.

Logistic regression models were used to estimate odds ratios of prevalent subclinical disease for each indicator of personal SES. Each indicator was then adjusted for: (1) sociodemographic characteristics (age, gender, and race); (2) behavioral characteristics (current and former smoking, alcohol intake, physical activity); and (3) biological characteristics (SBP, DBP, BMI, serum cholesterol, HDL, history of diabetes, blood glucose, use of anti-hypertensive medication). This same series of logistic regression analyses was conducted for the neighborhood socioeconomic summary score. Additional logistic regression analyses relating the neighborhood socioeconomic summary score to prevalent subclinical disease adjusted for personal SES indicators were also conducted. Virtually identical results were obtained after accounting for any potential withinneighborhood correlation in outcomes usingmodels with a random neighborhood intercept, so results for the simpler models are reported.

These analyses were based on the updated CHS database, which incorporates minor corrections, including genetic exclusions, if any, through 2001. Analyses were conducted using the SAS statistical software package, version 8.2 (Cary, N.C.). All participants gave written informed consent and all study protocols were 
approved by the Institutional Review Boards of participating institutions.

\section{Results}

Characteristics of the study sample are presented in Table 1. The mean (SD) age of the sample included in these analyses was $72.4(5.4)$ years. Just over $38 \%$ were male and $15.9 \%$ were Black. Sixty percent had at least one indicator of subclinical disease. Nearly $25 \%$ had an average family income less than $\$ 12,000$ and $25 \%$ had an average family income of $\$ 35,000$ or greater. Over $55 \%$ completed high school or less and just over $20 \%$ completed college or more. Blue-collar jobs (farming or forestry or craftsman, machine operator, or laborer) were reported by $16.9 \%$ of the sample. The mean blockgroup characteristics for participants in the sample were $\$ 32,309$ for median household income, $79.8 \%$ for blockgroup percent with complete high school, and $28.4 \%$ for block-group percent with complete college. The mean overall block-group score was 2.5 and ranged from -10.7 to 16.7 .

Nearly $13 \%$ of the sample currently smoked cigarettes and $26.8 \%$ reported using anti-hypertensive medication. Mean (SD) SBP was 136.4 (21.5) $\mathrm{mmHg}$ and mean (SD) DBP was $71.5(11.1) \mathrm{mmHg}$. Total serum cholesterol was elevated at $212.5(38.6) \mathrm{mg} / \mathrm{dl}$, while HDL averaged $56.0(15.9) \mathrm{mg} / \mathrm{dl}$.

As compared to those without prevalent subclinical disease at baseline, those with subclinical disease had significantly lower education, personal income, and neighborhood scores and were more likely to have blue-collar jobs (Table 1). They were also older and more likely to be male, Black, current smokers, and users of anti-hypertensive medications. Those with subclinical disease had higher SBP and fasting plasma glucose levels and lower HDL levels. They also reported less physical activity than those without subclinical disease.

Results from logistic regression relating personal and area SES indicators and subclinical disease are presented in Table 2. In models adjusted for age, gender, and race, those in the lowest income group ( $<\$ 12,000$ per year) had a $50 \%$ higher odds of having subclinical disease when compared with those in the highest group $(\geqslant \$ 35,000$ per year) $(\mathrm{OR}=1.50,95 \%$ CI $1.21-1.86)$. Similarly, those in the lowest education group (less than high school) had a $41 \%$ higher odds of having subclinical disease when compared with those in the highest group (completed college or graduate school) $(\mathrm{OR}=1.41,95 \%$ CI 1.18-1.69). Blue-collar workers also had significantly greater odds of disease compared with white-collar workers $(\mathrm{OR}=1.29,95 \%$ CI 1.05-1.59). Persons in the least wealthy tertile of block-group score had a $39 \%$ greater odds of subclinical disease compared with those in the most wealthy tertile $(\mathrm{OR}=1.39,95 \%$ CI 1.16-1.67), and those living in the second tertile also were at increased risk $(\mathrm{OR}=1.21,95 \%$ CI 1.02-1.43).

Adjustment for behavioral covariates (current and former smoking, alcohol consumption, physical activity) did not impact these relationships. After additional adjustment for biological covariates (BMI, SBP, DBP, total serum cholesterol, HDL, fasting plasma glucose, history of diabetes, and use of anti-hypertensive medication), only higher income remained significantly protective (Table 2). After adjustment for behavioral and biological risk factors, the association with subclinical disease prevalence was reduced in the lowest group relative to the highest group for income $(\mathrm{OR}=1.27 ; 95 \%$ CI 1.01-1.61), education $(\mathrm{OR}=1.18$; $95 \%$ CI $0.98-1.43)$, and block-group score $(\mathrm{OR}=1.22$; $95 \%$ CI 1.00-1.48). The association with subclinical disease was reduced by $34 \%$ for blue-collar occupation $(\mathrm{OR}=1.19 ; 95 \%$ CI 0.96-1.49).

Results from logistic regression relating area SES indicators and subclinical disease adjusted for personal SES factors and CVD risk factors are presented in Table 3. While point estimates suggest that persons in the lowest tertile of BGScore were more likely to have prevalent subclinical disease than those in the highest tertile even after adjustment for personal socioeconomic indicators and cardiovascular risk factors, none of the estimates achieved statistical significance. Personal income remained significantly inversely related to prevalent subclinical diseaseafter adjustment for neighborhood score, education, and occupation $(\mathrm{OR}=1.45$; 95\% CI 1.09-1.93 for the lowest relative to the highest groups), but this association was attenuated and became non-statistically significant in the model including behavioral and biomedical risk factors $(\mathrm{OR}=1.27$; 95\% CI 0.92-1.76). Having a blue-collar job was also significantly associated with increased risk of subclinical disease after adjustment for income, education, and block-group score $(\mathrm{OR}=1.29 ; 95 \%$ CI 1.03-1.62), but was also attenuated after risk-factor adjustment $(\mathrm{OR}=1.10 ; 95 \%$ CI $0.85-1.42)$.

\section{Discussion}

The relationship between CHD and personal SES has been established (Kaplan \& Keil, 1993). Other data suggest that neighborhood-level SES also impacts CHD risk, independent of personal SES (Diez-Roux et al., 2001b). It is less clear whether socioeconomic conditions are related to subclinical atherosclerosis and CVD in older adults. Our findings show that older persons with subclinical disease had lower education and household income and were more likely to have blue-collar jobs compared with those without prevalent subclinical disease. Further, older persons with subclinical disease 
Table 1

Means (SD) and percent distributions of socioeconomic status indicators and cardiovascular disease risk factors for entire sample and by subclinical disease prevalence

\begin{tabular}{|c|c|c|c|c|}
\hline & $\begin{array}{l}\text { Entire sample } \\
(N=3545)\end{array}$ & $\begin{array}{l}\text { No subclinical } \\
\text { disease }(N=1419)\end{array}$ & $\begin{array}{l}\text { Subclinical disease } \\
(N=2126)\end{array}$ & $p$-value ${ }^{\mathrm{a}}$ \\
\hline \multicolumn{5}{|l|}{ Demographic variables } \\
\hline Age (years) & $72.4(5.4)$ & $71.0(4.6)$ & $73.3(5.7)$ & $<0.001$ \\
\hline Male $(\%)$ & 38.3 & 32.4 & 42.3 & $<0.001$ \\
\hline Black $(\%)$ & 15.9 & 14.1 & 17.1 & 0.020 \\
\hline \multicolumn{5}{|l|}{ Subclinical disease status } \\
\hline Any subclinical disease $(\%)$ & 60.0 & $\mathrm{n} / \mathrm{a}$ & $\mathrm{n} / \mathrm{a}$ & $\mathrm{n} / \mathrm{a}$ \\
\hline \multicolumn{5}{|l|}{ Personal SES variables } \\
\hline Income $(\%)$ & & & & $<0.001$ \\
\hline$<\$ 12,000$ & 24.4 & 20.0 & 27.3 & \\
\hline$\$ 12,000-\$ 34,999$ & 51.0 & 52.8 & 49.9 & \\
\hline$\geqslant \$ 35,000$ & 24.6 & 27.2 & 22.8 & \\
\hline Education $(\%)$ & & & & $<0.001$ \\
\hline High school or less & 55.3 & 51.1 & 58.0 & \\
\hline Some college or vocational training & 23.3 & 25.3 & 21.9 & \\
\hline $\begin{array}{l}\text { Complete college or graduate } \\
\text { school }\end{array}$ & 21.5 & 23.6 & 20.1 & \\
\hline Blue-collar job (\%) & 16.9 & 13.5 & 18.9 & $<0.001$ \\
\hline \multicolumn{5}{|l|}{ Neighborhood SES variables } \\
\hline $\begin{array}{l}\text { Block group score (range }-10.7 \text { to } \\
16.7 \text { ) }\end{array}$ & 2.5 & $2.9(5.0)$ & $2.2(5.1)$ & $<0.001$ \\
\hline Median value of housing units $(\$)$ & $103,965(61,476)$ & $107,843(63,643)$ & $98,831(59,861)$ & \\
\hline Median household income (\$) & $32,248(14,997)$ & $33,131(15,053)$ & $31,658(14,749)$ & \\
\hline Adults completing high school (\%) & 79.8 & 80.9 & 79.7 & \\
\hline Adults completing college $(\%)$ & 28.4 & 30.0 & 27.4 & \\
\hline $\begin{array}{l}\text { Households receiving interest income } \\
(\%)\end{array}$ & 45.7 & 47.5 & 44.6 & \\
\hline $\begin{array}{l}\text { Employed persons in managerial/ } \\
\text { professional jobs }(\%)\end{array}$ & 33.0 & 33.6 & 31.2 & \\
\hline \multicolumn{5}{|l|}{ Cardiovascular disease risk factors } \\
\hline Smoking status $(\%)$ & & & & $<0.001$ \\
\hline Never smokers & 47.7 & 52.5 & 44.6 & \\
\hline Former smokers & 39.5 & 38.2 & 40.4 & \\
\hline Current smokers & 12.7 & 9.3 & 15.1 & \\
\hline Users of anti-hypertensive meds (\%) & 36.8 & 29.7 & 41.6 & $<0.001$ \\
\hline Alcohol intake (drinks/week) & $2.8(6.8)$ & $2.7(6.7)$ & $2.8(6.9)$ & 0.574 \\
\hline Physical activity (Kcal/week) & $1771(2039)$ & $1919(2093)$ & $1680(2003)$ & $<0.001$ \\
\hline Body mass index $\left(\mathrm{kg} / \mathrm{m}^{2}\right)$ & $26.6(4.7)$ & $26.5(4.8)$ & $26.7(4.5)$ & 0.432 \\
\hline Diastolic blood pressure $(\mathrm{mmHg})$ & $71.5(11.1)$ & $71.7(10.6)$ & $71.3(11.4)$ & 0.267 \\
\hline Systolic blood pressure (mmHg) & $136.4(21.5)$ & $131.9(19.3)$ & $139.34(22.3)$ & $<0.001$ \\
\hline Total serum cholesterol (mg/dl) & $212.5(38.6)$ & $211.3(35.9)$ & $213.5(40.1)$ & 0.098 \\
\hline HDL cholesterol (mg/dl) & $56.0(15.9)$ & $58.1(16.0)$ & $54.7(15.7)$ & $<0.001$ \\
\hline Glucose $(\mathrm{mg} / \mathrm{dl})$ & $108.8(34.7)$ & $104.2(25.6)$ & $111.8(39.3)$ & $<0.001$ \\
\hline
\end{tabular}

\footnotetext{
${ }^{a} p$-value for difference between those with and without prevalent subclinical disease.
} 
Table 2

Odds ratios of subclinical disease prevalence by categories of personal socioeconomic indicators and area socioeconomic characteristics

\begin{tabular}{|c|c|c|c|}
\hline & \multicolumn{3}{|c|}{ Odds ratio $(95 \% \mathrm{CI})$} \\
\hline & Model 1 & Model 2 & Model 3 \\
\hline \multicolumn{4}{|l|}{ Income } \\
\hline$<\$ 12,000$ & $\begin{array}{l}1.50 \\
(1.21-1.86)\end{array}$ & $\begin{array}{l}1.44 \\
(1.16-1.81)\end{array}$ & $\begin{array}{l}1.27 \\
(1.01-1.61)\end{array}$ \\
\hline$\$ 12,000-\$ 34,999$ & $\begin{array}{l}1.11 \\
(0.94-1.33)\end{array}$ & $\begin{array}{l}1.12 \\
(0.94-1.33)\end{array}$ & $\begin{array}{l}1.02 \\
(0.85-1.22)\end{array}$ \\
\hline$>\$ 35,000$ & 1.0 & 1.0 & 1.0 \\
\hline \multicolumn{4}{|l|}{ Education } \\
\hline High school or less & $\begin{array}{l}1.41 \\
(1.18-1.69)\end{array}$ & $\begin{array}{l}1.38 \\
(1.16-1.65)\end{array}$ & $\begin{array}{l}1.18 \\
(0.98-1.43)\end{array}$ \\
\hline Some college or vocational training & $\begin{array}{l}1.13 \\
(0.92-1.39)\end{array}$ & $\begin{array}{l}1.11 \\
(0.90-1.36)\end{array}$ & $\begin{array}{l}1.01 \\
(0.81-1.25)\end{array}$ \\
\hline Complete college or graduate school & 1.0 & 1.0 & 1.0 \\
\hline Blue-collar job & $\begin{array}{l}1.29 \\
(1.02-1.59)\end{array}$ & $\begin{array}{l}1.26 \\
(1.01-1.55)\end{array}$ & $\begin{array}{l}1.19 \\
(0.96-1.49)\end{array}$ \\
\hline \multicolumn{4}{|l|}{ Block group score } \\
\hline Tertile 1 & $\begin{array}{l}1.39 \\
(1.16-1.67)\end{array}$ & $\begin{array}{l}1.40 \\
(1.16-1.69)\end{array}$ & $\begin{array}{l}1.22 \\
(1.00-1.48)\end{array}$ \\
\hline Tertile 2 & $\begin{array}{l}1.21 \\
(1.02-1.43)\end{array}$ & $\begin{array}{l}1.22 \\
(1.03-1.45)\end{array}$ & $\begin{array}{l}1.15 \\
(0.96-1.37)\end{array}$ \\
\hline Tertile 3 & 1.0 & 1.0 & 1.0 \\
\hline
\end{tabular}

Model 1 adjusted for age, gender, and race.

Model 2 adjusted for covariates in Model 1 plus current and former smoking, alcohol intake, and physical activity.

Model 3 adjusted for covariates in Model 2 plus BMI, SBP, DBP, total serum cholesterol, HDL cholesterol, fasting blood glucose, ADA diabetes status, use of anti-hypertensive medication.

Table 3

Odds ratios of subclinical disease prevalence by area socioeconomic status to subclinical disease, adjusted for personal socioeconomic indicators and risk factors

\begin{tabular}{llll}
\hline & \multicolumn{2}{l}{ Odds ratio $(95 \% \mathrm{CI})$} \\
\cline { 2 - 4 } & Model 1 & Model 2 & Model 3 \\
\hline Block group score & & & \\
Tertile 1 & 1.19 & 1.25 & 1.15 \\
Tertile 2 & $(0.94-1.49)$ & $(0.97-1.60)$ & $(0.88-1.50)$ \\
Tertile 3 & 1.06 & 1.12 & 1.12 \\
& $(0.86-1.30)$ & $(0.90-1.39)$ & $(0.89-1.41)$ \\
\hline
\end{tabular}

Model 1 adjusted for education, income, blue-collar occupation.

Model 2 adjusted for covariates in Model 1 plus age, gender, race.

Model 3 adjusted for covariates in Model 2 plus current and former smoking, alcohol intake, physical activity, BMI, SBP, DBP, total serum cholesterol, HDL cholesterol, fasting blood glucose, ADA diabetes status, use of anti-hypertensive medication. were more likely than those without to live in neighborhoods with lower overall socioeconomic scores. Adjustment for behavioral and biological factors, which may partly mediate SES differences, diminished the strength of these associations, but did not fully explain the relationships. While neighborhood score appears to be inversely related to prevalent subclinical disease, especially for those in the highest tertile compared with those in the lowest, these associations were no longer statistically significant after adjustment for personal SES.

There are relatively few studies that examine the impact of personal SES on subclinical atherosclerosis, with the majority of those assessing carotid IMT (DiezRoux et al., 1995; Lynch et al., 1995, 1997; Lamont et al., 2000; Rosvall et al., 2000). Overall, SES appears to be related to IMT, but it remains unclear whether the association between personal SES and subclinical CVD is explained by traditional CVD risk factors. Among Finnish men aged 42-60 years, there were significant inverse relationships between education, income, and lifetime occupation and IMT, but the magnitude of risk 
was greatly diminished with adjustment for CVD risk factors (Lynch et al., 1995). However, additional analyses excluding men with prevalent CVD showed that the effect sizes persisted after risk-factor adjustment, as did the effect of SES on progression of IMT (Lynch et al., 1997). Analyses from the ARIC study found that, among persons without prevalent CVD, associations of personal socioeconomic indicators with IMT were greatly attenuated after adjustment for cardiovascular risk factors (Diez-Roux et al., 1995). In our analyses of elderly men and women without prevalent CVD, associations of personal and neighborhood SES with subclinical disease were also attenuated after adjustment for behavioral and biological covariates, although differences by personal income and education remained statistically significant.

In interpreting risk-factor adjusted results, it is important to emphasize that these risk factors are likely to be mediators rather than confounders of socioeconomic differences. Even if the risk-factors studied wholly mediate the socioeconomic differences, there are several reasons why associations of socioeconomic indicators with subclinical disease could persist after adjustment for established cardiovascular risk factors. Measurement error in the risk factors themselves is a possibility. In addition, in the elderly, risk-factor measures late in life may not adequately capture exposure to these risk factors over the lifecourse, and long-term exposures may be particularly relevant because atherosclerotic disease develops gradually over time. It may also be that SES differences in subclinical disease are mediated through processes that do not involve traditional risk factors, such as neurohormonal or biochemical reactions to stress. However, the interpretation of the persistence of associations after adjustment for risk factors as evidence of other mechanisms needs to be done cautiously given the limitations of estimating "direct effects" by adjusting for known mediators (Robins \& Greenland, 1992). Differences in the precision with which both outcomes and risk factors are measured may also explain differing results in the literature regarding the extent to which SES differences in subclinical disease do or do not persist after riskfactor adjustment. In addition, the persons included in the current analyses are a selected group in that they have avoided clinically manifest CVD well into the seventh decade of life and beyond. It is possible that in this group, standard risk factors are less strongly related to subclinical disease. It is also possible that crosssectional analyses reflect a downward drift of ill persons into low SES groups, although this is unlikely when subclinical disease (as opposed to clinically manifest disease) is the outcome.

There is little or no information relating SES (at either the neighborhood or individual-level) to indicators of subclinical disease other than carotid IMT. Whitehall II study investigators reported an inverse association between employment grade and both angina prevalence and electrocardiogram abnormalities (Marmot et al., 1991). Another study found lower likelihood of positive response to Rose angina among those being served at a large public hospital treating a mostly low SES clientele compared with those treated at a middle class HMObased hospital, but only among White subjects and not among Latino or Black subjects (Haywood, Ell, Sobel, de Guzman, \& Blumfield, 1993). In the Healthy Women Study, education was inversely related to EBCTassessed coronary and aortic calcification among postmenopausal women asymptomatic for CVD (Gallo, Matthews, Kuller, Sutton-Tyrrell, \& Edmundowicz, 2001). Our measure of subclinical disease is a composite of several measures, including IMT, ECG abnormalities, and Rose angina scores. Further analyses should be done in the CHS to assess whether particular aspects of subclinical disease are more affected by socioeconomic position than other indicators. The high prevalence of subclinical disease in this elderly cohort may also have hampered our ability to detect associations with socioeconomic factors.

There have been no previously reported findings relating neighborhood SES to subclinical disease. We found a strong trend across levels of neighborhood characteristics for the likelihood of subclinical disease. Again, behavioral risk factors did not account for the relationship, although adjustment for biological factors reduced the relationship somewhat. Although an inverse trend was still apparent, associations of neighborhood score with subclinical disease were no longer statistically significant after adjustment for personal socioeconomic indicators.

On the other hand, personal SES continued to be related to subclinical disease after accounting for neighborhood SES. This was true for income, even though the limitations of income as a marker of socioeconomic position in elderly adults has been noted (Robert \& House, 1996). There was no evidence in our data that associations of neighborhood characteristics with disease were especially strong in the elderly, or that neighborhood SES was more strongly related to disease than personal SES in this age group. However, our findings regarding the absence of statistically significant neighborhood differences after adjustment for personal socioeconomic indicators need to be interpreted with caution due to the gross misspecification of neighborhood characteristics, even when compared to personal income. Measurement error in neighborhood characteristics is likely to be especially important when a single measure pertaining to place of residence in old age (as opposed to a lifecourse measure) is used.

An important limitation of our study is its crosssectional nature. However, it is unlikely that the 
socioeconomic exposures that we investigated are the consequence of subclinical disease. The limitations of using block groups as proxies for neighborhoods have been noted (Diez-Roux, 2001) and arise from the fact that block-groups are likely to be very imperfect proxies for true "neighborhoods". In addition, both personal and neighborhood characteristics were assessed in old age and may be poor proxies of SES and neighborhood trajectories over the lifecourse which would be the most relevant factors to examine in relation to subclinical disease in the elderly. Wealth may also provide a better assessment of personal SES than income, which can substantially decline after retirement. Although there is a range of values for education, occupation, etc., the CHS cohort is known to be healthier and more educated than the general population of older adults from which the cohort was drawn. This may have made it more difficult to detect associations of personal and neighborhood characteristics with subclinical disease.

Our results show that socioeconomic advantage is inversely associated with the burden of subclinical CVD in old age. The patterning of subclinical disease by socioeconomic factors in the elderly strongly suggests that differences in cumulative exposures over the lifecourse, which are patterned by SES, are related to the development of atherosclerosis. Further work is needed to determine the processes through which socioeconomic factors over the lifecourse shape the development of cardiovascular risk.

\section{Acknowledgements}

Wake Forest University School of Medicine: Gregory L. Burke M.D. Wake Forest University_ECG Reading Center: Pentti M. Rautaharju M.D., Ph.D. University of California, Davis: John Robbins M.D. M.H.S. The Johns Hopkins University: Linda P. Fried M.D., M.P.H. The Johns Hopkins University-MRI Reading Center: Nick Bryan M.D., Ph.D., Norman J. Beauchamp M.D. University of Pittsburgh: Lewis H. Kuller, M.D., Dr.P.H. University of California, Irvine-Echocardiography Reading Center (baseline): Julius M. Gardin M.D. Georgetown Medical Center-Echocardiography Reading Center (follow-up): John S. Gottdiener M.D. New England Medical Center, BostonUltrasound Reading Center: Daniel H. O'Leary M.D. University of Vermont - Central Blood Analysis Laboratory: Russell P. Tracy Ph.D. University of Arizona, Tucson-Pulmonary Reading Center: Paul Enright M.D. Retinal Reading Center-University of Wisconsin: Ronald Klein M.D. University of Washington-Coordinating Center: Richard A. Kronmal Ph.D. NHLBI Project Office: Jean Olson, M.D., M.P.H.

\section{References}

Bots, M. L., Hoes, A. W., \& Koudstaal, P. J., et al. (1997). Common carotid intima-media thickness and risk of stroke and myocardial infarction: The Rotterdam study. Circulation, 96, 1432-1437.

Chambless, L. E., Heiss, G., \& Folsom, A. R., et al. (1997). Association of coronary heart disease incidence with carotid arterial wall thickness and major risk factors: The atherosclerosis risk in communities (ARIC) study, 1987-1993. American Journal of Epidemiology, 146, 483-494.

Diez-Roux, A. V. (2001). Investigating neighborhood and area effects on health. American Journal of Public Health, 91, 1783-1789.

Diez Roux, A. V., Kiefe, C. I., \& Jacobs, D. R., et al. (2001a). Area characteristics and individual-level socioeconomic position indicators in three population-based epidemiologic studies. Annals of Epidemiology, 11, 395-405.

Diez-Roux, A. V., Nieto, F. J., Tyroler, H. A., Crum, L. D., \& Szklo, M. (1995). Social inequalities and atherosclerosis. The atherosclerosis risk in communities study. American Journal of Epidemiology, 141, 960-972.

Diez-Roux, A. V., Stein Merkin, S., \& Arnett, D., et al. (2001b). Neighborhood of residence and incidence of coronary heart disease. New England Journal of Medicine, 345, 99-106.

Feldman, J. J., Makuc, D. M., \& Kleinman, J. C., et al. (1989). National trends in educational differentials in mortality. American Journal of Epidemiology, 129, 919-933.

Fried, L. P., Borhani, N. O., \& Enright, P., et al. (1991). The cardiovascular health study: Design and rationale. Annals of Epidemiology, 1, 263-276.

Gallo, L. C., Matthews, K. A., Kuller, L. H., Sutton-Tyrrell, K., \& Edmundowicz, D. (2001). Educational attainment and coronary and aortic calcification in postmenopausal women. Psychosomatic Medicine, 63, 925-935.

Haywood, L. J., Ell, K., Sobel, E., de Guzman, M., \& Blumfield, D. (1993). Rose Questionnaire responses among black, Latino, and white subjects in two socioeconomic strata. Ethnicity \& Disease, 3, 303-314.

Hodis, H. N., Mack, W. J., \& LaBree, L., et al. (1998). The role of carotid arterial intima-media thickness in predicting clinical coronary events. Annals of Internal Medicine, 128, 262-269.

Kaplan, G. A., \& Keil, J. E. (1993). Socioeconomic factors and cardiovascular disease: A review of the literature. Circulation, 88, 1973-1998.

Kuller, L., Borhani, N. O., \& Furberg, C., et al. (1994). Prevalence of subclinical atherosclerosis and cardiovascular disease and association with risk factors in the cardiovascular health study. American Journal of Epidemiology, 139, 1164-1179.

Lamont, D., Parker, L., \& White, M., et al. (2000). Risk of cardiovascular disease measured by carotid intima-media thickness at age 49-51: Lifecourse study. British Medical Journal, 320, 273-278.

Lynch, J., Kaplan, G. A., Salonen, R., Cohen, R. D., \& Salonen, J. T. (1995). Socioeconomic status and carotid atherosclerosis. Circulation, 92, 1786-1792.

Lynch, J., Kaplan, G. A., Salonen, R., \& Salonen, J. T. (1997). Socioeconomic status and progression of carotid athero- 
sclerosis: Prospective evidence from the Kuopio heart disease risk factor study. Arteriosclerosis Thrombosis and Vascular Biology, 17, 513-519.

Marmot, M. G., Smith, G. D., \& Stansfeld, S., et al. (1991). Health inequalities among British civil servants: The Whitehall II study. Lancet, 337, 1387-1393.

O’Leary, D. H., Polak, J. F., \& Kronmal, R. A., et al. (1999). Carotid-artery intima and media thickness as a risk factor for myocardial infarction and stroke in older adults. Cardiovascular health study collaborative research group. New England Journal of Medicine, 340, 14-22.

Robert, S., \& House, J. S. (1996). SES differentials in health by age and alternative indicators of SES. Journal of Aging and Health, 8, 359-388.
Robins, J. M., \& Greenland, S. (1992). Identifiability and exchangeability for direct and indirect effects. Epidemiology, 3, 143-155.

Rose, G., \& Marmot, M. G. (1981). Social class and coronary heart disease. British Heart Journal, 45, 13-19.

Rosvall, M., Ostergren, P. O., Hedblad, B., Isacsson, S. O., Janzon, L., \& Berglund, G. (2000). Occupational status, educational level, and the prevalence of carotid atherosclerosis in a general population sample of middle-aged Swedish men and women: Results from the Malmo diet and cancer study. American Journal of Epidemiology, 152, 334-346.

Salonen, J. T., \& Salonen, R. (1993). Ultrasound B-mode imaging in observational studies of atherosclerotic progression. Circulation, 87(suppl 3), II56-II65. 